Benchmarks

\title{
Fluorogenic "click" reaction for labeling and detection of DNA in proliferating cells
}

Kai Li ${ }^{1,2}, \mathrm{~L}$. Andrew Lee ${ }^{2}$, Xiaobing Lu ${ }^{1}$, and Qian Wang ${ }^{2}$

${ }^{1}$ State Key Laboratory of Fine Chemicals, Dalian University of Technology (DUT), Dalian, China, and ${ }^{2}$ Department of Chemistry and Biochemistry and Nanocenter, University of South Carolina, Columbia, SC, USA

BioTechniques 49:525-527 (July 2010) doi 10.2144/000113463

Keywords: 5-ethynyl-2'-deoxyuridine; cell proliferation; fluorogenic reaction; Cu-catalyzed alkyne-azide cycloaddition; click chemistry

A thymidine analog, 5-ethynyl-2'-deoxyuridine (EdU), has been reported as a rapid labeling tool for direct measurement of cells in S-phase. The alkynyl group of EdU is a biologically inert group that undergoes an extremely selective reaction with azido-functionalized groups via $\mathrm{Cu}(\mathrm{I})$-catalyzed alkyneazide cycloaddition (CuAAC or "click") reaction. Here we report the highly efficient reaction of the terminal alkynyl group of EdU with a pro-fluorogenic compound, 3-azido-7-hydroxycoumarin, to afford an intense fluorescent 1,2,3-triazole product, which occurs only after the $\mathrm{CuAAC}$ reaction. This new method eliminates concerns for residual fluorescence since the unreacted precursors are optically inactive. The procedure therefore does not require extensive wash steps to remove the unreacted fluorescent dyes in the sample, allowing for immediate quantification and visualization after the reaction. The advantage over currently available commercial products is its potential to streamline high-throughput applications and help minimize errors.

The detection of cell proliferation with 5-bromo-2'-deoxyuridine (BrdU) and $3 \mathrm{H}$-labeled nucleotides has been broadly used for short-term labeling studies to probe DNA synthesis and cell proliferation rates, as well as the progression of $S$ phase with various cell types (1). Compared with the other thymidine analogs, 5-ethynyl-2'deoxyuridine (EdU) can be considered as an ideal alternative reagent since it does not require harsh DNA denaturing conditions such as with BrdU, or necessitate radiolabeling followed by cumbersome detection methods (2-6). The free alkynyl group on EdU can be readily detected through its reaction with probes bearing azido groups in a $\mathrm{Cu}(\mathrm{I})$-catalyzed alkyne-azide cycloaddition (CuAAC) reaction, also known as "click" reaction (7). Unlike bulky antibodies, alkyne and azide groups are very small in size and have a particularly narrow distribution of reactivity, ability to conveniently attach to organic compounds, and are quite indifferent to solvent and $\mathrm{pH}$. Therefore, the $\mathrm{CuAAC}$ reaction has been ideal for linking biomolecules in complex environments, such as within cells, with high efficiency and chemoselectivity.

In our previous studies, we have developed the water-soluble, pro-fluorogenic dye 3-azido-7-hydroxycoumarin, which is a non-fluorescent compound until the dye reacts with terminal alkynes to afford an intense fluorescent 1,2,3-triazole product (8). This fluorogenic reaction has proved to be a versatile tool in the application of protein labeling, cell imaging, and combinatorial synthesis of fluorescence dyes due to the high reaction efficiency at mild conditions $(8-10)$. Furthermore, both azide and alkyne groups are biologically inert, giving this reaction much potential in bioimaging applications. Here we report the application of the dye in the rapid labeling and detection of DNA in proliferating cells, that activates only upon reacting with its specific DNA analog (Figure 1).

EdU and coumarin were synthesized and purified as described previously $(8,11)$. Both chemicals were relatively simple to synthesize and purify in large quantities, making them attractive and inexpensive alternatives to $\mathrm{BrdU}$ and anti-BrdU antibody labeling reagents. The incorporation of EdU to the DNA backbone of proliferative cells was examined based on the concentration of EdU $(10$ and $50 \mu \mathrm{M})$ and time (from $30 \mathrm{~min}$ to $24 \mathrm{~h}$ ) (Figure $2, A$ and B). Three different cell linesHeLa, NIH-3T3, and KB - were selected for cell proliferation analyses (Figure 2C). To all of these cell lines, EdU showed no toxicity even after incubating the cells with $50 \mu \mathrm{M}$ for $24 \mathrm{~h}$. Since the cells were not treated to synchronize their cell division, a short incubation time ( $30 \mathrm{~min}$ ) with EdU or BrdU labeled $35 \%$ of the cells with the thymidine analogs. Upon increasing the incubation time $(>12 \mathrm{~h}$ ), we found nearcomplete labeling of cells with $50 \mu \mathrm{M} \mathrm{EdU}$ (94\%, $P<0.05)$, and only partial labeling at the lower concentration (75\% with 10 $\mu \mathrm{M}$ EdU). For short incubation periods $(<12 \mathrm{~h})$, both concentrations were sufficient to label the cells actively replicating its genetic material. However, at longer incubation time periods, the higher concentration of EdU was necessary to obtain a higher loading.

It isimportant to note that this fluorogenic CuAAC reaction is highly efficient (near completion within $1 \mathrm{~h}$ at room temperature) and can be used in a quantitative manner via cell sorter or a fluorimeter. In addition, only the final 1,2,3-triazole product yields a fluorescent signal $(8-10,12)$. The CuAAC reaction affords superior regioselectivity and quantitative transformation under extremely mild conditions. A standard DNA proliferation assay requires a permeabilization step to allow the large anti-BrdU antibodies to penetrate the cell and nuclear membranes. Harsh treatment with $\mathrm{HCl}$ is also required to denature the nucleic acids in order to expose the antigenic sites. Furthermore, additional wash steps are required to remove unbound, free fluorescent dyes, but can result in loss of sample and signal. The dye reported here is small enough to penetrate directly into the nucleus, which circumvents the permeabilization, denaturation, and subsequent wash steps. In comparison to previous reports $(4,5)$, there are two distinct improvements over the use of commercially available fluorescein dyes. Since the unreacted precursors emit no fluorescence and only the final 1,2,3- 


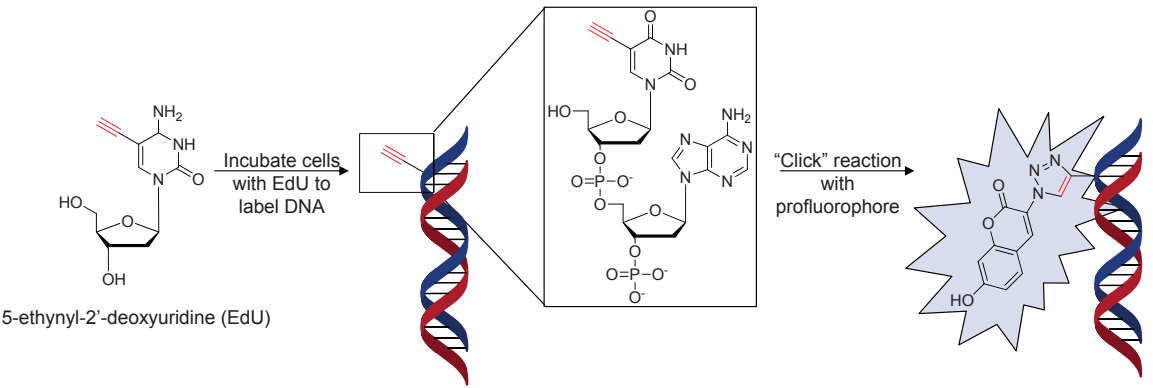

Figure 1. Labeling of DNA with EdU followed by a fluorogenic "click" reaction. Detection of incorporated nucleotide analog is achieved via reaction of the ethynyl group with a pro-fluorogenic dye, 3-azido-7-hydroxycoumarin. Standard BrdU assays and conventional fluorescent dye labeling of EdU require additional washing steps to remove the unbound fluorescent dyes. In our protocol, these extra wash steps are unnecessary, as the precursor is nonfluorescent until the conjugation between coumarin and $\mathrm{EdU}$ is achieved. In brief, the cells are incubated with $\mathrm{EdU}(10 \mu \mathrm{M}$ or $50 \mu \mathrm{M})$ at $37^{\circ} \mathrm{C}, 5 \% \mathrm{CO}_{2}$ for $30 \mathrm{~min}$ to $24 \mathrm{~h}$ in complete media (MEM/EBSS supplemented with $10 \%$ fetal bovine serum, $1 \times$ penicillin, and $1 \times$ streptomycin). Then the EdU-labeled cells were fixed with $4 \%$ paraformaldehyde for $10 \mathrm{~min}$, washed with PBS, and then reacted with $50 \mu \mathrm{M}$ dye in buffer solution (100 mM Tris- $\mathrm{HCl}$ pH 8.0 , $100 \mathrm{mM} \mathrm{L}$-ascorbic acid, $1 \mathrm{mM} \mathrm{CuSO}_{4}$ ) at room temperature for $1 \mathrm{~h}$.

A

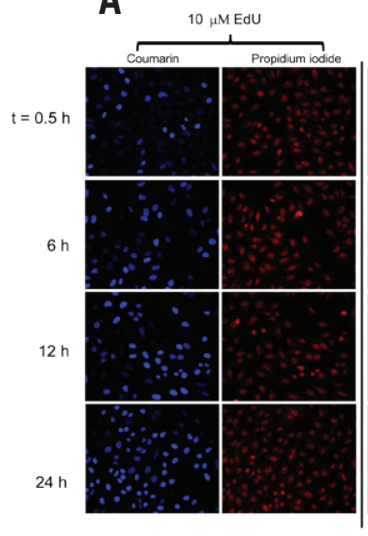

$50 \mu \mathrm{M} \mathrm{EdU}$

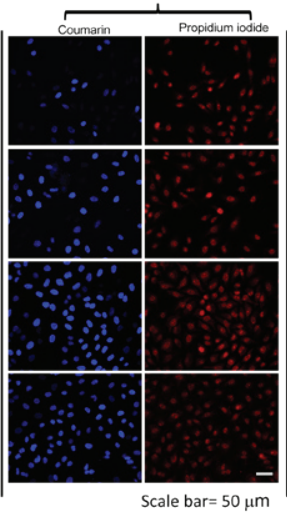

B

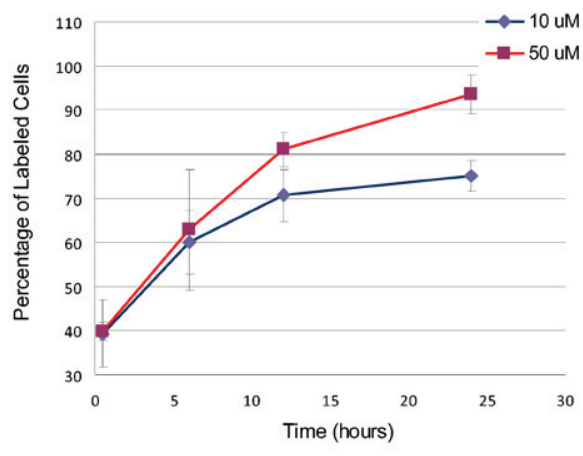

C

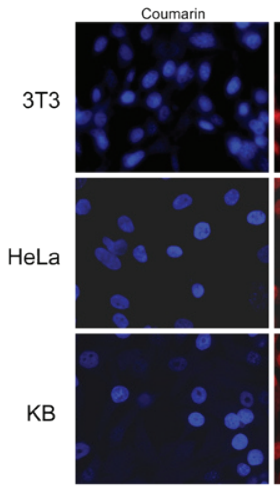

Scale bar $=25 \mu \mathrm{m}$

Figure 2. "Clicking on" fluorescence in proliferating cells. (A) Fluorescent microscopy images of cells labeled with EdU and reacted with coumarin, and counterstained nuclei with propidium iodide. The cells were seeded on No. 2 coverslips and allowed to adhere to the glass covers overnight. The media was supplemented for the allotted time, after which the cells were fixed with $4 \%$ paraformaldehyde for 10 min, washed with PBS, and then reacted with $50 \mu \mathrm{M}$ dye (100 mM Tris- $\mathrm{HCl} \mathrm{pH} \mathrm{8.0,} 100 \mathrm{mM}$ L-ascorbic acid, $1 \mathrm{mM} \mathrm{CuSO}_{4}$ ) at room temperature for $1 \mathrm{~h}$. After the reaction, propidium iodide (red) was added to the sample for $10 \mathrm{~min}$. To obtain the best images, the cells on coverslips are rinsed once in water to remove excess salts and placed on glass slides with a PVA/glycerol mounting solution. The samples were examined with an Olympus IX 86 confocal microscope using Semrock DAPI and Cy3 filters. EdU $(50 \mu \mathrm{M})$ incorporation (blue) gave near complete labeling of cellular DNA after $24 \mathrm{~h}$ (images processed with Image Pro Plus V 6.0). (B) Cell labeling efficiency against incubation time and concentration. For short incubation periods, there was no difference in labeling efficiencies for the two different concentrations, but at longer incubation periods, a higher concentration of EdU was necessary to label all of the cells $(P<0.05, n>200)$. Cells were counted from four image panels taken randomly with the fluorescent microscope. (C) Three different cell lines were incubated with $50 \mu \mathrm{M}$ EdU and reacted with azido-coumarin, and counterstained with propidium iodide.

triazole product yields a signal, the signals are attributed only to the labeled DNA. The elimination of the three or four wash steps (5-10 min each) reduces not only the time to process the sample, but decreases the chance for sample and signal loss. One major concern for the azido-coumarin dye is its poor stability in oxidative conditions, and prolonged storage in DMSO or DMF is not permissible. Future variants with improved storage and tailored emission spectra will likely expand the potential of pro-fluorogenic dyes (13).

Incubation of another fluorogenic dye developed in our lab, anthracene azide, (14) resulted in the dye intercalating into the DNA and labeling the cells regardless of the alkynyl groups. Biotin azide, used in an attempt to quantify the cell numbers using avidin-horseradish peroxidase (HRP), had been a poor choice due to the high concentration of biotin in many of these tumor cell lines. While it was possible to quench the intracellular biotin with excess avidin followed by blocking with biotin, these experimental procedures would involve additional steps and reagents to block the intracellular biotin followed by additional washing to remove excess biotin and avidin. These additional steps would increase the amount of time needed to process each sample. Instead, a direct conjugation of HRP to EdU may be more ideally suited.

In summary, a fluorogenic $\mathrm{CuAAC}$ reaction was introduced for labeling and detection of DNA in proliferating cells.
Thymidine analog incorporation to the DNA of proliferative cells was characterized based on concentration (10 and $50 \mu \mathrm{M} \mathrm{EdU}$ ) and time (30 min to $24 \mathrm{~h}$ ). The profluorophore 3-azido-7-hydroxycoumarin eliminates the need for repetitive wash steps, which are required for other labeling processes with dye-labeled antibodies and fluorescein azide.

\section{Acknowledgments}

Funding was provided in part by the US National Science Foundation CAREER Program, US Department of Defense Breast Cancer Research Program, the Alfred P. Sloan Scholarship, the Camille Dreyfus Teacher Scholar Award, and the 
University of South Carolina Nanocenter; as well as the China Scholarship Council (to K.L.).

\section{Competing interests}

The authors declare no competing interests.

\section{References}

1. Leif, R.C., J.H. Stein, and R.M. Zucker. 2004 A short history of the initial application of anti$5-\mathrm{BrdU}$ to the detection and measurement of $\mathrm{S}$ phase. Cytometry A 58:45-52.

2. Chehrehasa, F., A.C.B. Meedeniya, P. Dwyer, G. Abrahamsen, and A. MackaySim. 2009. EdU, a new thymidine analogue for labelling proliferating cells in the nervous system. J. Neurosci. Methods 177:122-130.

3. Diermeier-Daucher, S., S.T. Clarke, D. Hill, A. Vollmann-Zwerenz, J.A. Bradford, and G. Brockhoff. 2009. Cell type specific applicability of 5-ethynyl-2'-deoxyuridine (EdU) for dynamic proliferation assessment in flow cytometry. Cytometry A 75:535-546.

4.Salic, A. and T.J. Mitchison. 2008 . A chemical method for fast and sensitive detection of DNA synthesis in vivo. Proc. Natl. Acad. Sci. USA 105:2415-2420.

5. Buck, S.B., J. Bradford, K.R. Gee, B.J. Agnew, S.T. Clarke, and A. Salic. 2008. Detection of
S-phase cell cycle progression using 5-ethynyl2 '-deoxyuridine incorporation with click chemistry, an alternative to using 5-bromo2'-deoxyuridine antibodies. BioTechniques 44:927-929.

6. Jao, C.Y. and A. Salic. 2008. Exploring RNA transcription and turnover in vivo by using click chemistry. Proc. Natl. Acad. Sci. USA 105:15779-15784.

7. Rostovtsev, V.V., L.G. Green, V.V. Fokin, and K.B. Sharpless. 2002. A stepwise Huisgen cycloaddition process: copper(I)-catalyzed regioselective "ligation" of azides and terminal alkynes. Angew. Chem. Int. Ed. Engl. 41:25962599.

8. Sivakumar, K., F. Xie, B.M. Cash, S. Long, H.N. Barnhill, and Q. Wang. 2004. A fluorogenic 1,3-dipolar cycloaddition reaction of 3 -azidocoumarins and acetylenes. Org. Lett. 6:4603-4606

9. Beatty, K.E., J.C. Liu, F. Xie, D.C. Dieterich, E.M. Schuman, Q. Wang, and D.A. Tirrell. 2006. Fluorescence visualization of newly synthesized proteins in mammalian cells. Angew. Chem. Int. Ed. Engl. 45:7364-7367.

10. Beatty, K.E., F. Xie, Q. Wang, and D.A. Tirrell. 2005. Selective dye-labeling of newly synthesized proteins in bacterial cells. J. Am. Chem. Soc. 127:14150-14151.

11. Meneni, S., I. Ott, C.D. Sergeant, A. Sniady, R. Gust, and R. Dembinski. 2007. 5-Alkynyl-2'-deoxyuridines: chromatography-free synthesis and cytotoxicity evaluation against human breast cancer cells. Bioorg. Med. Chem. 15:3082-3088.
12.Seela, F., V.R. Sirivolu, and P. Chittepu. 2008. Modification of DNA with octadiynyl side chains: Synthesis, base pairing, and formation of fluorescent coumarin dye conjugates of four nucleobases by the alkyne-azide "click" reaction. Bioconjug. Chem. 19:211224.

13. Le Droumaguet, C., C. Wang, and Q. Wang. 2010. Fluorogenic click reaction. Chem. Soc. Rev. 39:1233-1239.

14.Xie, F., K. Sivakumar, Q. Zeng, M.A. Bruckman, B. Hodges, and Q. Wang. 2008. A fluorogenic 'click' reaction of azidoanthracene derivatives. Tetrahedron 64:2906-2914.

Received 26 October 2009; accepted 28 May 2010

Address correspondence to Qian Wang, Department of Chemistry and Biochemistry and Nanocenter, University of South Carolina, Columbia, SC, 29208, USA. e-mail:wang@mail. chem.sc.edu.

\section{BioTechnioues \\ The International Journal of Life Science Methods}

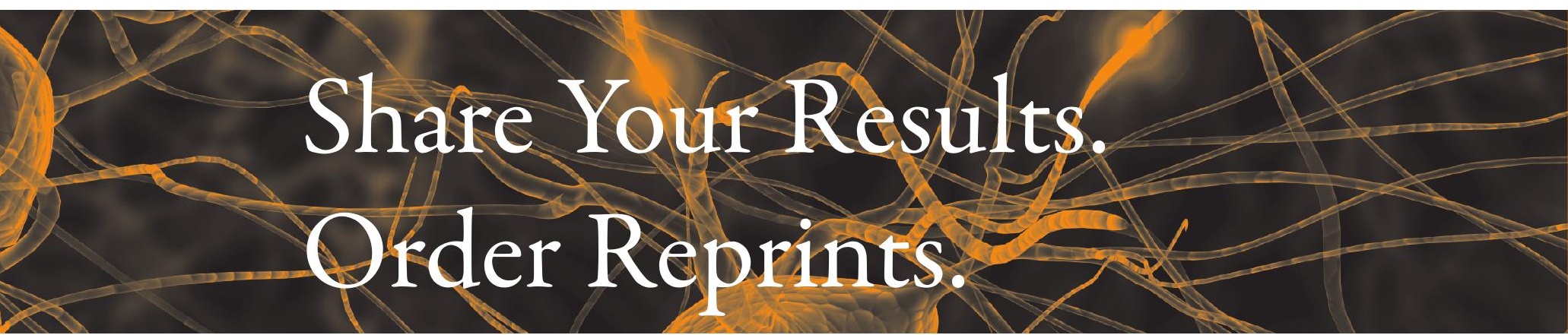

Author Reprints

Make the most of your hard work by ordering reprints of your article published in BioTechniques. Reprints are an inexpensive and easy way to distribute your findings to students and colleagues alike.

Corporate Reprints

Leverage BioTechniques, the most powerful brand in the market. Reprints help support your sales effort by utilizing articles that spotlight your brand/products to educate customers at meetings and industry trade events. 